CORRECTION

\title{
Correction: Long non-coding RNA DLEU1 predicts poor prognosis of gastric cancer and contributes to cell proliferation by epigenetically suppressing KLF2
}

Xiaobin Li, Zongze Li, Ziwen Liu, Jianchun Xiao, Shuting Yu and Yimin Song

(c) The Author(s), under exclusive licence to Springer Nature America, Inc. 2021

Cancer Gene Therapy (2022) 29:873; https://doi.org/10.1038/s41417-021-00374-w

Correction to: Cancer Gene Ther 25, 58-67; https://doi.org/10.1038/ s41417-017-0007-9; published online 27 December 2017

After publication it was discovered that there was an error in Figure $3 \mathrm{E}$. This unintentional mistake occurred during image editing/composition. The authors' repeated the experiment, and sorted out the original data of the experiments related to the wrong pictures. They found that there was an error in the insertion of the Figure 3E. They have replaced the correct images and the correct Figure 3E is included with this correction.
The flow cytometry results of the authors repeated experiments show that down-regulation of DLEU1 can really promote the apoptosis of AGS cells and BGC-823 cells, which is consistent with the original conclusion.

The original article has been corrected.
AGS

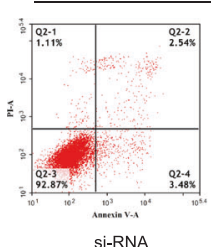

Si-RNA

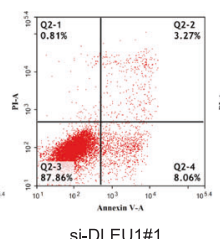

Si-DLEU1\#1

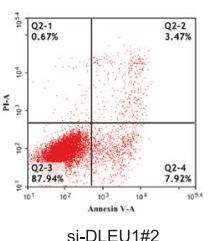

Si-DLEU1\#2

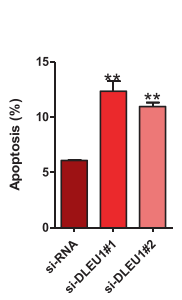

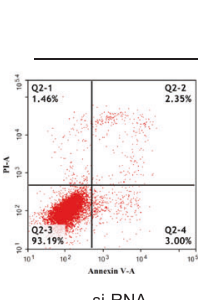

si-RNA
BGC-823

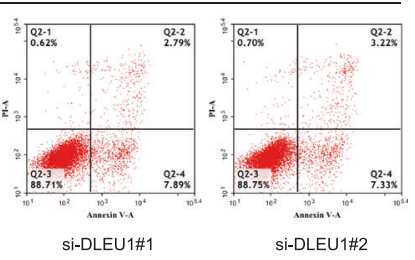

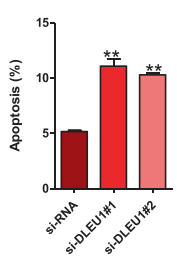

